\title{
Produção de leite de ovelhas Hampshire Down e Ile de France até os 84 dias de lactação
}

\author{
Milk production of Hampshire Down and Ile de \\ France ewes up to 84 days of lactation
}

\author{
Marcos Rodrigues Podleskis ${ }^{1}$; Edson Luis de Azambuja Ribeiro ${ }^{2 *}$; \\ Marco Antonio da Rocha ${ }^{3}$; Leandro das Dores Ferreira da Silva ${ }^{4}$; \\ Ivone Yurika Mizubuti ${ }^{3}$; Rinaldo Masato Mori ${ }^{5}$; Danilo Otavio \\ Laurenti Ferreira ${ }^{6}$; Tiago Rodrigues Casimiro ${ }^{6}$
}

\section{Resumo}

Este trabalho teve como objetivo avaliar a produção de leite de ovelhas das raças Hampshire Down e Ile de France, com 6 ou 8 dentes e amamentando machos ou fêmeas, bem como verificar o efeito do número de cordeiros sobre a produção de leite de ovelhas Hampshire Down. O experimento foi conduzido na Fazenda Escola da Universidade Estadual de Londrina, no período de junho a dezembro de 2001. A alimentação dos animais consistiu de pastagem de grama Coastcross (Cynodon dactylon (L.) Pers), sendo que no período de menor oferta e qualidade da pastagem (Junho a Setembro), as ovelhas receberam diariamente suplementação com $2 \mathrm{~kg}$ de silagem de milho e $100 \mathrm{~g}$ de milho grão triturado. As estimativas de produção de leite foram realizadas através de ordenha manual, após aplicação de 3 UI de ocitocina, aos 14, 28, 42, 56, 70 e 84 dias de lactação. Não houve diferenças $(P>0,05)$ para a produção de leite e para pesos das ovelhas entre as raças envolvidas, entre as idades das ovelhas e entre ovelhas Hampshire Down amamentando um ou dois cordeiros. Porém, em relação ao sexo dos cordeiros, ovelhas amamentando machos tiveram uma produção média diária de leite significativamente $(\mathrm{P}<0,05)$ maior do que ovelhas amamentando fêmeas $(1121,98 \mathrm{vs} 886,27 \mathrm{ml})$. A produção de leite foi decrescente dos 14 aos 84 dias de lactação. Concluiu-se que as duas raças avaliadas apresentaram produções de leite similar, porém ovelhas amamentando machos produziram mais leite.

Palavras-chave: Curva de lactação, habilidade materna, ovinos, pesos, raças

1 Médico Veterinário, Mestre.

2 Zootecnista, PhD, Bolsista CNPq, Professor da Universidade Estadual de Londrina, CCA, Departamento de Zootecnia, Caixa Postal 6001,86051-970, Londrina, Paraná, e-mail: elar@uel.br.*

3 Médico Veterinário(a), Doutor(a), Professores da UEL, CCA, Departamento de Zootecnia.

4 Zootecnista, Doutor, Professor da UEL, CCA, Departamento de Zootecnia.

5 Médico Veterinário, Doutorando em Ciência Animal, UEL.

6 Alunos do curso de Medicina Veterinária, UEL.

* Autor para correspondência. 


\begin{abstract}
The objective of this study was to evaluate the milk production of Hampshire Down and Ile de France ewes, six or eight teeth old, nursing male or female lambs, as well as to evaluate the effect of litter size on milk production of Hampshire Down ewes. This study was carried out at the Farm School of the State University of Londrina, Brazil, between June and December, 2001. The animals fed on a Coastcross (Cynodon dactilon (L) Pears) pasture, and received food supplementation daily, $2 \mathrm{~kg}$ of corn silage and $100 \mathrm{~g}$ of grounded corn, from June to September. Milk production was estimated, by hand milking the ewes with the aid of $3 \mathrm{IU}$ of oxitocyn injection, at days 14, 28, 42, 56, 70 and 84 of lactation. There were no differences $(\mathrm{P}>0.05)$ in milk production and in ewe weights between breeds, ages, and between Hampshire Down ewes nursing twins or singlets. But, ewes nursing male lambs had greater $(\mathrm{P}<0.05)$ daily milk production than ewes nursing female lambs $(1121,98 \mathrm{vs} 886,27 \mathrm{ml})$. Milk production decreased from day 14 up to day 84 of lactation. It was concluded that the evaluated breeds had similar milk production, and that ewes nursing male lambs had greater milk production.
\end{abstract}

Key words: Breeds, lactation curve, maternal ability, sheep, weight

\section{Introdução}

O leite produzido pelas ovelhas principalmente, nas primeiras semanas de vida, é a principal fonte de alimentação para o cordeiro. Durante o primeiro mês de vida, cerca de $64 \%$ do crescimento do cordeiro está associado a produção de leite da ovelha (MOTTA et al., 2000). A ovelha produz $80 \%$ do leite de uma lactação nas primeiras oito semanas e em condições normais, após a décima segunda semana, a produção de leite é mínima, e o leite da ovelha supre menos de $10 \%$ das necessidades nutricionais do cordeiro (COIMBRA FILHO, 1997).

A produção de leite é influenciada por vários fatores, entre estes podem ser citados: o peso vivo da ovelha, o número de cordeiros, a idade da ovelha e a nutrição. Durante muito tempo os estudos foram feitos comparando-se a produção de leite associada a variável raça, determinando-se a produção de leite em diferentes raças, em igualdade de condições. Quando se considerou o peso vivo, observou-se que a produção estava relacionada mais a esta característica do que as diferenças raciais (MINOLA; GOYENECHEA, 1975).

Para Snowder e Glimp (1991), o número de cordeiros mamando é um fator de grande importância na produção de leite. Cardellino e Benson (1994) e Ramsey, Hatfield e Wallace (1998) relataram que ovelhas que criaram gêmeos produziram mais leite do que as que criaram apenas um cordeiro. Após revisarem os trabalhos de vários autores Gardner e Hogue (1964) verificaram que diferentes teorias foram postuladas para as diferenças na produção de leite por ovelhas com cordeiros simples em oposição a ovelhas com gêmeos. A habilidade dos gêmeos em esvaziar completamente as glândulas mamárias no início da lactação tem sido apontada como o principal fator. Apesar da maioria dos pesquisadores encontrar uma maior produção de leite em ovelhas amamentando cordeiros gêmeos, alguns encontraram resultados diferentes. Ramsey, Hatfield e Wallace (1994) e Godfrey, Gray e Collins (1997) verificaram que o número de cordeiros nascidos não afetou a produção de leite.

Wohlt et al. (1984) verificaram que o sexo da prole não influiu na produção de leite, sendo que as fêmeas e os machos consumiram quantidades similares de leite durante as oito semanas de lactação. Por outro lado, a produção de leite aumenta até a quarta lactação, a partir da qual diminui (MINOLA; GOYENECHEA, 1975). Cardellino e Benson (1994) encontraram efeitos significantes para idade da ovelha, sendo maior para as ovelhas de dois anos em comparação com ovelhas de um ano, resultado semelhante foi observado por Motta et al. (2000).

A lactação é o período onde a ovelha apresenta o maior requerimento de nutrientes para o ciclo de produção anual. Susin (1996) afirmou que as ovelhas normalmente perdem peso durante o início da 
lactação. A magnitude desta perda varia, dependendo da qualidade e quantidade de alimento disponível, do número de cordeiros amamentados, de fatores ambientais e do potencial produtivo da ovelha.

O presente trabalho teve por finalidade, avaliar a produção de leite de ovelhas das raças Hampshire Down e Ile de France, com 6 ou 8 dentes e amamentando machos ou fêmeas, bem como verificar o efeito do número de cordeiros sobre a produção de leite de ovelhas Hampshire Down.

\section{Material e Métodos}

O experimento foi conduzido na Fazenda Escola da Universidade Estadual de Londrina, Londrina, Paraná. Segundo a classificação de Köppen, Londrina tem característica climática Cfa - clima subtropical com verões quentes, geadas pouco freqüentes e tendência de concentração das chuvas nos meses de verão, contudo sem estação seca definida. A umidade relativa é de 75 a $80 \%$, com média de precipitação pluviométrica e temperatura média para os meses de agosto, setembro, outubro e novembro, respectivamente, de $62,5 \mathrm{~mm}$ e $18,5^{\circ} \mathrm{C}$, 112,5 e $19,5^{\circ} \mathrm{C}, 137,5 \mathrm{~mm}$ e $21,5^{\circ} \mathrm{C}$ e $162,5 \mathrm{~mm}$ e $23,5^{\circ} \mathrm{C}$ (IAPAR, 2002).

Foram utilizadas 32 ovelhas, de 6 a 8 dentes e suas crias, distribuídas em dois grupamentos genéticos. Destas, vinte e três ovelhas eram Hampshire Down, sendo 16 com cordeiros únicos (sete machos e nove fêmeas) e sete com cordeiros gêmeos, e nove ovelhas Ile de France com cordeiros únicos (três machos e seis fêmeas). Das ovelhas com cordeiros únicos 12 eram de 6 dentes e 13 de 8 dentes. Os animais foram mantidos sob condição de pastagem em uma área de 5 ha com Coastcross (Cynodon dactylon (L.) Pers), dividida em sete piquetes. Os nascimentos dos cordeiros ocorreram de 03 de agosto a 13 de setembro de 2001. Durante o período de menor oferta e qualidade da pastagem (Junho a Setembro), as ovelhas receberam suplementação com silagem de milho ( $\pm 2 \mathrm{~kg}$ /animal) e milho em grão triturado (100 g/animal) e sal mineral a vontade no cocho. No mês de agosto, a pastagem apresentou uma disponibilidade de $2.352 \mathrm{~kg}$ de MS/ ha, tendo a seguinte composição química (base seca), $\mathrm{PB}=8,7 \%, \mathrm{FDN}=88,1 \%$ e $\mathrm{FDA}=46,8 \%$, e $\mathrm{MS}=$ $52,0 \%$.

Para a estimativa de produção de leite, aos 14, $28,42,56,70$, e 84 dias de idade, os cordeiros foram separados de suas mães as $8 \mathrm{~h}$ e fechados em baias individuais do aprisco. As ovelhas foram pesadas às $9 \mathrm{~h}$ e novamente reunidas com seus cordeiros por um período de 30 minutos permitindo que mamassem para o completo esvaziamento do úbere. As 09:30 h as ovelhas foram então separadas de seus cordeiros e estes permaneceram estabulados separados de suas mães por um período de \pm 4 horas. A partir das 13:30 $\mathrm{h}$ as ovelhas foram ordenhadas. Para auxiliar a ejeção do leite foi administrada uma injeção de 3 UI de ocitocina por via intramuscular (LEMOS NETO; CUNHA, 1994). Em seguida, fez-se a ordenha manual da metade direita do úbere, e a quantidade coletada foi multiplicada por dois para ajustar a produção por animal, e com base no tempo de restrição de mamada do cordeiro, a produção foi ajustada para 24 horas.

A produção de leite e o peso corporal das ovelhas foram submetidos à análise de variância, utilizando o procedimento GLM (SAS, 1985). Para as ovelhas amamentando um cordeiro foram levados em consideração no modelo, as variáveis, grupo genético da ovelha, idade da ovelha, sexo do cordeiro e as interações duplas entre elas. $\mathrm{Na}$ análise incluindo o número de cordeiros amamentados, foram utilizados apenas os dados das ovelhas Hampshire Down, não sendo considerado nesse caso o efeito do sexo do cordeiro.

\section{Resultados e Discussão}

A análise de variância mostrou que as interações entre as variáveis independentes não foram significativas $(\mathrm{P}>0,05)$. As médias para os pesos das ovelhas segundo o grupo genético e a idade da ovelha e segundo o sexo do cordeiro são apresentadas na 
Tabela 1, onde pode ser observado que não houve diferença $(\mathrm{P}>0,05)$ para os pesos entre essas variáveis. Esses resultados estão de acordo com Ribeiro et al. (2000), que não encontraram diferenças nos pesos de borregos de 12 meses dos dois grupos genéticos testados. Em relação as idades, não ocorreram diferenças, provavelmente, pelas mesmas serem muito próximas. Contudo, Zeppenfeld et al. (2002) compararam ovelhas com idade variando entre 4 e 5 anos e borregas com a primeira cria aos 12 meses e também não encontraram diferenças significativas.

Tabela 1. Médias ( \pm erro padrão) para pesos de ovelhas $(\mathrm{kg})$ do parto ao $84^{\circ}$ dia de lactação de acordo com o grupo genético e a idade das ovelhas e o sexo dos cordeiros.

\begin{tabular}{|c|c|c|c|c|c|c|}
\hline \multirow[b]{2}{*}{ Dias de lactação } & \multicolumn{2}{|c|}{ Grupo genético } & \multicolumn{2}{|c|}{ Idade da ovelha } & \multicolumn{2}{|c|}{ Sexo do cordeiro } \\
\hline & $\begin{array}{c}\text { Hampshire } \\
\text { Down }\end{array}$ & Ile de France & 8 dentes & 6 dentes & Macho & Fêmea \\
\hline Ao parto & $56,25 \pm 1,90$ & $52,22 \pm 2,68$ & $56,02 \pm 2,45$ & $52,45 \pm 2,17$ & $53,47 \pm 2,69$ & $55,00 \pm 2,01$ \\
\hline 14 & $55,28 \pm 1,92$ & $53,13 \pm 2,70$ & $56,93 \pm 2,47$ & $51,49 \pm 2,19$ & $53,78 \pm 2,71$ & $54,63 \pm 2,03$ \\
\hline 28 & $54,54 \pm 2,06$ & $52,20 \pm 2,91$ & $55,00 \pm 2,66$ & $51,73 \pm 2,36$ & $52,24 \pm 2,92$ & $54,49 \pm 2,19$ \\
\hline 42 & $52,54 \pm 1,80$ & $50,62 \pm 2,54$ & $54,00 \pm 2,32$ & $49,17 \pm 2,06$ & $50,46 \pm 2,55$ & $52,71 \pm 1,91$ \\
\hline 56 & $54,30 \pm 2,11$ & $51,10 \pm 2,81$ & $54,38 \pm 2,57$ & $51,02 \pm 2,39$ & $51,36 \pm 2,81$ & $54,04 \pm 2,22$ \\
\hline 70 & $53,28 \pm 2,13$ & $50,31 \pm 3,01$ & $54,71 \pm 2,75$ & $48,89 \pm 2,43$ & $50,99 \pm 3,02$ & $52,61 \pm 2,26$ \\
\hline 84 & $52,42 \pm 2,26$ & $48,86 \pm 3,19$ & $54,66 \pm 2,92$ & $46,62 \pm 2,58$ & $50,26 \pm 3,20$ & $51,02 \pm 2,40$ \\
\hline
\end{tabular}

$(\mathrm{P}>0,05)$

De uma maneira geral, houve perda de peso do parto aos 42 dias de lactação, uma pequena recuperação dos 42 aos 56 dias, com perda de peso posterior. Estas mudanças de peso podem ser explicadas pelos requerimentos das ovelhas e pela alimentação disponível, onde nos primeiros 40 a 45 dias de lactação os requerimentos das ovelhas são bastante altos, e o volumoso e o concentrado oferecidos não supriram esses requerimentos. A partir dos 40 dias com a diminuição na produção de leite, o alimento fornecido foi suficiente para atender os requerimentos para produção de leite e para um pequeno ganho de peso. A partir dos 56 dias as ovelhas voltaram a perder peso pois a a alimentação suplementar (silagem de milho mais grão de milho) foi retirada e a pastagem não supriu as exigências nutricionais dos animais. Snowder e Glimp (1991) observaram que as ovelhas perderam peso do início até o pico (28 a 42 dias) da lactação, mas iniciaram a recuperação após o mesmo. Godfrey, Gray e Collins (1997) observaram que mesmo em ovelhas que não apresentaram um pico de produção de leite, onde a produção foi decrescente dos 7 aos 63 dias, que as mesmas perderam peso até os 21 a 35 dias de lactação, com pequeno ganho posterior.

$\mathrm{O}$ número de cordeiros amamentados não influenciou $(\mathrm{P}>0,05)$ o peso das ovelhas do parto aos 84 dias de lactação, onde ovelhas amamentando um cordeiro perderam em média $42 \mathrm{~g}$ por dia e as amamentando gêmeos, $107 \mathrm{~g}$ por dia (Tabela 2). Apesar de não haver diferença significativa para os pesos, observa-se que no início da lactação as ovelhas amamentando cordeiros gêmeos perderam mais peso do que as ovelhas amamentando cordeiros únicos. Isto ocorreu porque a necessidade total de leite para os cordeiros gêmeos é maior, e a ovelha lança mão de suas reservas corporais para atender essa maior exigência nutricional. Como pode ser visto (Tabela 4), apesar de não ter ocorrido diferença estatística $(\mathrm{P}>0,05)$, as ovelhas amamentando cordeiros gêmeos tiveram valores numericamente maiores para a produção de leite até os 28 dias de lactação. Estes resultados concordam com os encontrados por Snowder e Glimp (1991). 
Tabela 2. Médias ( \pm erro padrão) para pesos do parto ao $84^{\circ}$ dia de lactação em ovelhas Hampshire Down de acordo com o número de cordeiros nascidos e criados

\begin{tabular}{ccc}
\hline \multirow{2}{*}{ Dias de lactação } & \multicolumn{2}{c}{ Pesos (kg) } \\
\cline { 2 - 3 } & Únicos & Gêmeos \\
\hline Ao parto & $55,68 \pm 2,28$ & $55,36 \pm 6,67$ \\
14 & $54,66 \pm 2,25$ & $52,54 \pm 6,58$ \\
28 & $53,85 \pm 2,38$ & $48,06 \pm 6,96$ \\
42 & $52,21 \pm 2,08$ & $46,51 \pm 6,08$ \\
56 & $53,51 \pm 2,49$ & $44,65 \pm 7,16$ \\
70 & $52,92 \pm 2,39$ & $46,20 \pm 6,99$ \\
84 & $52,13 \pm 2,39$ & $46,36 \pm 6,99$ \\
\hline
\end{tabular}

$(\mathrm{P}>0,05)$
Em relação a produção de leite pode-se observar (Tabela 3) que não houve diferenças para grupo genético e idade das ovelhas, com médias gerais para a lactação, respectivamente, para ovelhas Hampshire Down e Ile de France de 1045,97 e 962,29 ml. Trabalhos comparando outras raças, que não as usadas nesse experimento, onde os pesos entre os grupos eram parecidos, também não encontraram diferenças nas produções de leite. As raças de corte Suffolk, Polypay, Columbia e Rambouillet foram comparadas por Snowder e Glimp (1991) e Godfrey et al (1997) compararam as raças Barbados Blackbelly, Nativa da Flórida e St. Croix White, e encontraram produções semelhantes entre as diferentes raças. Ramsey, Hatfield e Wallace (1998), também, não encontraram diferenças na produção de leite entre ovelhas Suffolk e Targhee.

Tabela 3. Médias ( \pm erro padrão) para produção de leite $(\mathrm{ml})$ de ovelhas do $14^{\circ}$ ao $84^{\circ}$ dia de lactação de acordo com o grupo genético e a idade das ovelhas e o sexo dos cordeiros

\begin{tabular}{ccccccc}
\hline \multirow{2}{*}{$\begin{array}{c}\text { Dias de } \\
\text { lactação }\end{array}$} & \multicolumn{2}{c}{ Grupo genético } & \multicolumn{2}{c}{ Idade da ovelha } & \multicolumn{2}{c}{ Sexo do cordeiro } \\
\cline { 2 - 7 } & Hampshire Down & Ile de France & 8 dentes & 6 dentes & Macho & Fêmea \\
\hline 14 & $1668,28 \pm 133,97$ & $1186,90 \pm 189,03$ & $1455,86 \pm 172,81$ & $1399,31 \pm 153,15$ & $1587,17 \pm 189,70$ & $1268,01 \pm 142,15$ \\
28 & $1358,31 \pm 92,14$ & $1305,66 \pm 129,99$ & $1441,03 \pm 118,85$ & $1222,93 \pm 105,33$ & $1411,84 \pm 130,46$ & $1252,13 \pm 97,76$ \\
42 & $1083,09 \pm 64,48$ & $1222,55 \pm 90,98$ & $1196,72 \pm 83,18$ & $1108,93 \pm 73,72$ & $1302,71 \pm 91,31 \mathrm{a}$ & $1002,93 \pm 91,30 \mathrm{~b}$ \\
56 & $960,30 \pm 86,44$ & $888,60 \pm 114,84$ & $842,40 \pm 105,04$ & $1006,51 \pm 97,73$ & $935,24 \pm 114,98$ & $913,67 \pm 90,98$ \\
70 & $581,11 \pm 57,40$ & $509,49 \pm 80,99$ & $540,95 \pm 74,04$ & $549,65 \pm 65,62$ & $583,24 \pm 81,28$ & $507,36 \pm 60,91$ \\
84 & $628,53 \pm 81,54$ & $677,01 \pm 115,04$ & $779,95 \pm 105,18$ & $525,59 \pm 93,21$ & $925,54 \pm 115,45 \mathrm{a}$ & $379,99 \pm 86,52 \mathrm{~b}$ \\
Média & $1045,97 \pm 46,78$ & $962,29 \pm 62,15$ & $1039,89 \pm 56,84$ & $968,37 \pm 52,89$ & $1121,98 \pm 62,23 \mathrm{a}$ & $886,27 \pm 49,24 \mathrm{~b}$ \\
\hline
\end{tabular}

$\mathrm{a}, \mathrm{b}(\mathrm{P}<0,05)$

A produção de leite foi semelhante entre as idades. De acordo com Minola e Goynechea (1975) a produção de leite aumenta até a quarta lactação, a partir da qual diminui. Motta et al. (2000) compararam a produção de leite entre borregas e ovelhas Texel e encontraram diferença significativa $(\mathrm{P}<0,05)$ para ovelhas de quatro anos, as quais produziram mais leite do que as borregas de dois anos durante a $3^{\mathrm{a}} \mathrm{e}$ $4^{\mathrm{a}}$ semanas de lactação. Resultado similar foi encontrado por Cardellino e Benson (1994) que compararam a produção de ovelhas de um ou dois anos de idade, e encontraram maiores produções $(\mathrm{P}<0,05)$ para as ovelhas mais velhas.
Em relação ao sexo do cordeiro, ovelhas amamentando machos produziram em média $26,6 \%$ mais leite do que ovelhas amamentando fêmeas (Tabela 3). De maneira geral, os resultados encontrados são diferentes dos citados por Wohlt et al. (1984) e Torres-Hernadez e Hohemboken (1980), que concluíram que o sexo do cordeiro não influenciou significativamente na produção de leite.

Não houve diferença $(\mathrm{P}>0,05)$ na produção de leite entre ovelhas amamentando um ou dois cordeiros (Tabela 4). Esse resultado discorda de Minola e Goyenechea (1975), que afirmaram que ovelhas que criam gêmeos produzem mais leite do 
que ovelhas que criam apenas um cordeiro. Cardellino e Benson (1994) e Ramsey, Hatfield e Wallace (1998) em seus experimentos encontraram que a produção de leite foi altamente significativa $(\mathrm{P}<0,001)$, sendo superior para ovelhas amamentando gêmeos em relação às ovelhas amamentando cordeiros únicos. Por outro lado Godfrey et al. (1997) não encontraram diferenças $(\mathrm{P}>0,1)$ para a produção de leite de acordo com o número de cordeiros mamando.

Tabela 4. Médias ( \pm erros padrão) para produção de leite (ml) do $14^{\circ}$ ao $84^{\circ}$ dia de lactação em ovelhas Hampshire Down de acordo com o número de cordeiros nascidos e criados

\begin{tabular}{ccc}
\hline Dias de lactação & Gêmeos & Únicos \\
\hline 14 & $1886,70 \pm 406,80$ & $1606,06 \pm 139,35$ \\
28 & $1688,66 \pm 295,01$ & $1371,45 \pm 101,05$ \\
42 & $672,97 \pm 213,40$ & $1091,34 \pm 73,10$ \\
56 & $563,10 \pm 258,12$ & $961,03 \pm 89,99$ \\
70 & $517,09 \pm 135,03$ & $604,29 \pm 46,26$ \\
84 & $214,82 \pm 192,30$ & $604,35 \pm 65,87$ \\
Média & $930,25 \pm 154,52$ & $1027,29 \pm 53,87$ \\
\hline
\end{tabular}

$(\mathrm{P}>0,05)$

Nas Tabelas 3 e 4 observa-se que houve um decréscimo da produção de leite durante o período de 14 a 84 dias de lactação. Os resultados encontrados na literatura mostram que a produção de leite em ovelhas criando um ou dois cordeiros apresentaram um pico de produção entre 14 e 28 dias de lactação (MINOLA; GOYENECHEA, 1975; TORRES-HERNADEZ; HOHEMBOKEN, 1980; ZEPPENFELD et al., 2002). Por outro lado, Ramsey, Hatfield e Wallace (1998) observaram um pico de lactação ao redor dos 50 dias após o parto. Snowder e Glimp (1991) que iniciaram as estimativas no $28^{\circ}$ dia de lactação, não observaram um pico de lactação, com produções decrescentes até o final da mesma.

\section{Conclusões}

Ovelhas Hampshire Down e Ile de France, com 6 e 8 dentes, e amamentando cordeiros simples ou gêmeos, tiveram produções de leite semelhantes. Porém, ovelhas amamentando cordeiros machos produziram mais leite do que ovelhas amamentando fêmeas.

\section{Referências}

CARDELLINO, R.A.; BENSON, M.E. Lactation curves of crossbred ewes as affected by rearing type an age of dam. Journal of Animal Science, Champaign, v.72, suppl. 1, p.307, 1994.

COIMBRA FILHO, A. Técnicas de criação de ovinos. 2.ed. Guaíba: Agropecuária, 1997. 102p.

GARDNER, R.W.; HOGUE, D.E. Effects of energy intake and number of lambs suckled on milk yield, milk composition and energetic efficiency of lactating ewes. Journal of Animal Science, Champaign, v.23, n.4, p.935942, 1964.

GODFREY, R.W.; GRAY, M.L.; COLLINS, J.R. Lamb growth and milk production of hair and wool sheep in a semi-arid tropical environment. Small Ruminant Research, Amsterdam, v.24, p.77-83, 1997.

IAPAR. Monitoramento agroclimático do Paraná. Disponível na Internet http://www.pr.gov.br/iapar/sma/ index.html. Acesso em 30 de outubro de 2002.

LEMOS NETO, M.J.; CUNHA, E.A. Comparação de métodos estimativos da produção de leite de ovelhas a pasto. Boletim de Indústria Animal, Nova Odessa, v.51, n.2, p.139-142, 1994.

MINOLA, J.; GOYENECHEA, J. Praderas \& Lanares: producion ovina en alto nível. Montevideo: Hemisferio Sur, 1975.365p.

MOTTA, O.S.; PIRES,C.C.; SILVA,J.H.S.; ROSA,G.T.;FULBER,M.; GARCIA,A.G.; Produção de leite das ovelhas e suas correlações com ganho de peso dos cordeiros. In: REUNIÃO ANUAL DA SOCIEDADE BRASILEIRA DE ZOOTECNIA, 37., 2000, Viçosa, MG. Anais... Viçosa: SBZ: 2000. p.107.

RAMSEY, W.S.; HATFIELD, P.G.; WALLACE, J.D; SOUTHWARD, G.M. Relationships among ewe milk production and ewe and lamb forage intake in Targhee ewes nursing single or twin lambs. Journal of Animal Science, Champaign, v.72, n.4, p.811-816, 1994. 
RAMSEY, W.S.; HATFIELD, P.G.; WALLACE, J.D. Relationships among ewe milk production and ewe and lamb forage intake in Suffolk and Targhee ewes nursing single or twin lambs. Journal of Animal Science, Champaign, v.76, n.4, p.1247-1253, 1998.

RIBEIRO, E.L.A.;ROCHA, M.A..; MIZUBUTI, I.Y.; MORI, R.M. Ganho de peso e componentes do peso vivo em borregos Ile de France inteiros ou castrados e Hampshire Down castrados abatidos aos doze meses de idade. Ciência Rural, Santa Maria, v.30, n.2, p.333-336, 2000.

SAS. SAS User's Guide: Statistics. 5.ed. Cary, NC, 1985. 956p.

SNOWDER, G.D.; GLIMP, H.A. Influence of breed, number of suckling lambs, and stage of lactation on ewe milk production and lamb growth under range conditions. Journal of Animal Science, Champaing, v.69, n.3, p.923930, 1997.

SUSIN, I. Exigências nutricionais de ovinos e estratégias de alimentação. In: . Nutrição de Ovinos. Jaboticabal: FUNEP, 1996. p.119-142.
TORRES-HERNANDEZ, G.; HOHENBOKEN, W. Relationships between ewe milk production and composition and preweaning lamb weight gain. Journal of Animal Science, Champaing, v.50, n.4, p.597-603, 1980.

WOHLT, J.E.; FOY, W.L.; KNIFFEN, D. M.; TROUT, J. R. Milk yield by Dorset ewes as affected by sibling status, sex and age of lamb, and measurement. Journal of Dairy Science, Champaign, v.67, n.4, p.802-807, Apr.1984.

WOHLT, J.E.;FOY, W.L.;KNIFFEN, D.M.;TROUT, J.R. Milk yield by Dorset ewes as affected by sibling status, sex and age of lamb, and measurement. Journal of Dairy Science, v.67, p.802-807, 1984.

ZEPPENFELD, C.C. et al. Produção de leite e consumo de ovelhas e borregas com primeira cria aos doze meses e ganho de peso dos filhos. In: REUNIÃO ANUAL DA SOCIEDADE BRASILEIRA DE ZOOTECNIA, 39., 2002, Recife, PE. Anais... Recife: SBZ, 2002. CD-ROM. 